\title{
Active Control of Blood Flow in the Aorta Using External Magnetic Field
}

\author{
N. Aggoune ${ }^{1}$, G. Mebarki ${ }^{2}$, M. Nezar ${ }^{3}$, M. S. Aggoune ${ }^{4}$, R. Abdessemed ${ }^{4}$ \\ ${ }^{1}$ LaMsM Laboratory, Department of Electrical Engineering, Faculty of Technology, University \\ of Batna 2, Batna 05000, Algeria \\ e-mail: n.aggoune@univ-batna2.dz \\ 2 LESEI Laboratory, Department of Mechanical Engineering, Faculty of Technology, \\ University of Batna 2, Algeria \\ e-mail: g.mebarki@univ-batna2.dz \\ ${ }^{3}$ LICECGS Laboratory, Department of Electrical Engineering, Faculty of Technology, \\ University of Batna 2, Algeria \\ e-mail: m.nezar@univ-batna2.dz \\ ${ }^{4}$ LEB Laboratory, Department of Electrical Engineering, Faculty of Technology, University of \\ Batna 2, Algeria \\ e-mail: ms.aggoun@univ-batna2.dz \\ e-mail: r.abdessemed@univ-batna2.dz \\ *corresponding author
}

\begin{abstract}
The objective of the present study is the active flow control of blood in the aorta with atherosclerosis using an External Magnetic Field (EMF) in order to facilitate the blood flow. For that purpose, a numerical investigation has been developed with a Magneto-hydrodynamics flow modelisation. The blood is considered homogeneous, incompressible and Newtonian and the fluid flow is assumed to be unsteady, two-dimensional and laminar. The aorta tissue is electrically conductive. Fluent software has been used to solve the governing equations. The results relating to velocity, pressure and the wall shear stress indicate that the presence of the EMF considerably influences the blood flow. The flow control deals with the effects of the EMF direction of application and its intensity. The results show that by applying an EMF, the blood velocity and pressure in the aorta are entirely affected. The direction and the intensity of the EMF allow minimization of the flow instabilities due to the geometrical singularities. Therefore, applying an EMF can be considered an appropriate method for flow control in order to obtain a uniform blood circulation around the atherosclerosis.
\end{abstract}

Keywords: Magneto-hydrodynamics, Blood flow, Finite volume method, External Magnetic Field, Atherosclerosis, Active control.

NOMENClATURE

$\begin{array}{llll}A & \text { magnetic potential vector } & R e & \text { Reynolds number } \\ B & \text { magnetic induction vector } & \mathrm{t} & \text { time } \\ D & \text { displacement vector } & \mathrm{u}, \mathrm{v} & \text { velocity vector components } \\ E & \text { electric field vector } & \mathrm{x}, \mathrm{y} & \text { spatial coordinates } \\ f & \text { Lorentz force vector } & \varepsilon & \text { electric permittivity }\end{array}$




$\begin{array}{ll}g & \text { gravity vector } \\ H & \text { magnetic field } \\ J & \text { electric current density } \\ L & \text { length } \\ P & \text { pressure }\end{array}$

$\begin{array}{ll}\mu & \text { magnetic permeability } \\ v & \text { cinematic viscosity } \\ \rho & \text { fluid density } \\ \sigma & \text { electric conductivity } \\ \omega & \text { angular frequency }\end{array}$

\section{Introduction}

The Magneto-hydrodynamics (MHD) is the study of the effect of the magnetic field on a conductive fluid. The electromagnetic fields applications have become more evident in our domestic, professional and medical environments. They interact with human body and this interaction depends strongly on their frequency and intensity and some short-term effects can be observed (Health Protection Agency 2008). Two control techniques have been used in the literature to control fluid flow. They can be classified as active or passive methods. The active control method involves external power to improve the blood flow, whereas the passive control method uses surface or geometrical modifications to the flow by incorporating inserts or additional devices.

Despite the numerous studies carried out on the effects of the electromagnetic field over the last few decades, there is no consensus in the scientific community on the negative effects as they are considered a double-edged weapon. While some argue that electromagnetic fields have negative implications (Yu and Shang 2014, Gungor et al. 2015), others, contrarily certify that they can be used for diagnosis such as in medical imaging (MRI) or for treatment of some cancer tumors by the technique of hyperthermia or in magneto therapy (Kanai and Taniguchi 2012). The modelling of the distribution of electromagnetic field in biological tissues must take into account the particularities of the studied system, such as the tissues electromagnetic properties, which constitutes a major difficulty, both in mathematical analysis and in numerical implementation (Siauve et al. 2003, Hoang et al. 2009, Ducreux et al. 2009, Frénéa-Robin 2009, Sharma et al. 2013).

Bio-magnetic fluid dynamics is a new area in fluid mechanics which deals with the dynamics of blood in the presence of magnetic field and its importance resides in the fact that it aims to find solutions, especially non-invasive, to certain diseases and conditions related to the human body. Bad blood circulation is an increasingly common problem in people's health and is considered to be the main cause of a large number of pathologies such as cardiovascular accidents. In order to enhance the blood flow parameters in the vessels, mainly viscosity and flow velocity, several investigations have been carried out using the effect of magnetic fields to reduce thickening, or viscosity of blood, which could be an alternative to drugs designed to ensure the fluidity of blood in humans (Alghamdi and El-Ghazaly 2012, Gmitrov 2014).

The results obtained by Tao and Huang (2011) demonstrate that blood viscosity could be controlled and maintained within the desired range by means of magnetic pulses appropriate intensity and duration. Indeed, their experiments showed that by applying a magnetic field parallel to the direction of the blood flow and of intensity equal to $1.3 \mathrm{~T}$, for about one minute, the blood viscosity decreases from 20 to $30 \%$, then increases slowly and regains its initial value after two or three hours. Also, experiments performed by Haik et al. (2001) showed that the blood flow decreases by $30 \%$ when subjected to a high magnetic field of $10 \mathrm{~T}$. The decrease in flow is due to an increase in the apparent viscosity of the blood due to the magnetic field. The effect of magnetic field on the biofluid has been presented by Mohammed (2016). He found that in the presence of a magnetic field, the viscosity of the blood decreases. The electrical conductivity of the blood which depends on its speed increases considerably. Tzirtzilakis and Loukopoulos (2005) studied the effects of the magnetic field on velocity, temperature field, skin friction 
andheat transfer rate of a biomagnetic fluid in a channel under the influence of a strong, stable and a uniform magnetic field applied locally. They demonstrate that the magnetic field influences considerably the flow field causing the formation of two vortices at the area of the two points where the influence of the magnetic field starts and stops.

Misra and Shit (2007) presented a numerical model of the effect of magnetic field on blood flow through different segments of the circulatory system with different diameters such as the aorta, femoral artery, carotid artery and coronary artery. Their results showed clearly that the magnetic field has the potential to reduce blood flow through arteries, wall shear stress and volumetric flow and it is also possible to reduce these amounts to any desired level by increasing or decreasing the magnetic field intensity. For that purpose, the authors suggest to use external magnetic field applications in the clinical treatment of hemodynamic diseases, such as hypertension. In the same context, a general mathematical model for blood flow simulations under strong non-uniform magnetic fields is presented by Kenjere (2008) and validated for different geometries, ranging from a single cylinder to the actual right coronary artery in humans. The temporal dependence of shear stress on the wall for different stenosis and the effects of nonuniform magnetic fields imposed on the blood flow model are analyzed. The results obtained can be used to study new strategies for optimizing strong non-uniform magnetic fields for localized cancer therapy because they create significant changes in flux characteristics, allowing this technique to be used to optimize targeted drug delivery (Aggoune et al. 2017).

A magneto hydrodynamic approach has been used by Shah et al. (2016) to study the behavior of blood flow with magnetic particles distributed uniformly through a circular cylindrical tube subjected to a magnetic field applied perpendicular to its direction. The obtained results show that the velocity of the blood as well as that of the magnetic particles decreases by increasing the magnetic field. This same approach has been used experimentally by Sharma and al. (2015) to measure the velocity of artificial blood with different magnetic fields intensities. The model results show that the velocity of blood and magnetic particles is appreciably reduced under the influence of magnetic field.

The effect of an external magnetic field is also used by Parmar et al. (2013) to control the blood velocity and the shear stress on the wall of the artery in the presence of overlapping stenosis. The authors demonstrated that it is possible to reduce these parameters to any desired level by increasing or decreasing the intensities of the field, which will constitute a new medical protocol in the treatment of cardiovascular diseases such as hypertension and atherosclerosis. Atherosclerosis is a deposit by accumulation of different elements (fat, blood, fibrous tissue) on part of the internal wall of an artery. The diameter of the artery decreases, which can obstruct the blood circulation. Its complications are chronic and cumulative, progressing slowly and could cause the formation of a thrombus that will slow or stop blood flow quickly, resulting in necrosis of living tissue.

In this study, a numerical simulation has been performed in order to investigate the effect of an external magnetic field (EMF) on the blood behaviour throughout the aorta, especially when encountering obstacles such as atherosclerosis. A solution of the complete Magnetohydrodynamic Navier-Stokes equations was obtained using Fluent software. The aim of this work is to control the blood flow in the human aorta. The flow control deals with the effects of the EMF direction and intensity. 


\section{Mathematical formulation}

Blood exhibits magneto-hydrodynamic (MHD) properties as it is an electrically conducting fluid. To study the effect of the applied magnetic field on blood behaviour, a simulation was performed to solve the Maxwell and Navier-Stokes coupled equations.

\subsection{Magneto static modelling}

When magnetic field is applied perpendicularly to the direction of blood flow, the velocity of blood tends to decrease due to the existence of Lorentz force. This force is produced on interaction of electric and magnetic fields. When solving a magnetic or electrical problem, it is often useful to use the appropriate potentials instead of the fields. The partial differential equations obtained using the Maxwell equations in terms of magnetic vector potential (A) are adopted in the present work. The conservation of magnetic flux is given by:

$$
\overrightarrow{\operatorname{Rot}} \vec{B}=0
$$

where $\vec{B}$ is the magnetic induction vector.

The Ampere's law is given by:

$$
\overrightarrow{R o t} \vec{H}=\vec{J}+(\partial \vec{D} / \partial t)
$$

$\vec{H}, \vec{J}$ and $\vec{D}$ are respectively the magnetic field, the electric current density and the displacement vector.

The Lorentz law specifies that:

$$
\overrightarrow{R o t} \vec{E}=-(\partial \vec{B} / \partial t)
$$

where $\vec{E}$ is the electric field vector.

The constituent relations for the materials are given by:

$$
\begin{aligned}
& \vec{J}=\sigma \vec{E} \\
& \vec{D}=\varepsilon \vec{E} \\
& \vec{B}=\mu \vec{H}
\end{aligned}
$$

where $(\sigma)$ is the electric conductivity, $(\varepsilon)$ is the electric permittivity and $(\mu)$ is the magnetic permeability.

These equations are simplified using the potential vector $\vec{A}$ :

$$
\vec{B}=\overrightarrow{\operatorname{Rot}} \vec{A}
$$

Equation (2) will be rewritten as follows:

$$
\overrightarrow{R o t} \vec{B}=\mu \vec{J}+\mu \varepsilon(\partial \vec{E} / \partial t)=\mu \sigma \vec{E}+\mu \varepsilon(\partial \vec{E} / \partial t)
$$

In harmonic regime, Eq. (8) becomes:

$$
\overrightarrow{\operatorname{Rot}} \vec{B}=\mu(\sigma+j \omega \varepsilon) \vec{E}
$$

With $(\omega)$ is the angular frequency of the applied signal. 
If the operator $(\overrightarrow{R o t})$ is introduced on both sides of Eq. (9), the result will be written as follows:

$$
\begin{gathered}
\overrightarrow{\operatorname{Rot}}(\overrightarrow{\operatorname{Rot} \vec{B}})=\mu(\sigma+j \varepsilon \omega) \overrightarrow{\operatorname{Rot} \vec{E}}=\mu(\sigma+j \varepsilon \omega)(-j \omega \vec{B})=-\mu\left(j \omega \sigma-\varepsilon \omega^{2}\right) \vec{B} \\
\overrightarrow{\operatorname{Rot}}(\overrightarrow{\operatorname{Rot} B})=-\mu\left(j \omega \sigma-\varepsilon \omega^{2}\right) \overrightarrow{\operatorname{Rot}} \vec{A}
\end{gathered}
$$

Then,

$$
\overrightarrow{\operatorname{Rot} \vec{B}}=-\mu\left(j \omega \sigma-\varepsilon \omega^{2}\right) \vec{A}
$$

Finally, the equation of the magneto static model can be reduced to:

$$
\left(j \omega \sigma-\varepsilon \omega^{2}\right) \vec{A}+\vec{\nabla} \wedge((1 / \mu) \vec{\nabla} \wedge \vec{A})=0
$$

\subsection{Fluid dynamics modelling}

The governing equations of the computational fluid dynamics analysis imply mass and momentum conservation equations. For unsteady two dimensional, laminar and incompressible fluid flow, these equations are given by:

Continuity equation:

$$
\vec{\nabla}(\rho \vec{v})=0
$$

Momentum equation:

$$
(\vec{v} \vec{\nabla}) \vec{v}=-(1 / \rho) \vec{\nabla} p+v \nabla^{2} \vec{v}+\vec{g}+\vec{f}
$$

Where $(\rho)$ is the blood density, $(v)$ is the blood cinematic viscosity and $\vec{v}$ is the blood velocity vector.

$\vec{f}$ is the Lorentz force vector, expressed by:

$$
\vec{f}=\vec{J} \wedge \vec{B}
$$

with,

$$
\vec{J}=\sigma(\vec{E}+\vec{v} \wedge \vec{B})
$$

then,

$$
\vec{f}=\sigma \vec{E} \wedge \vec{B}+\sigma(\vec{v} \wedge \vec{B}) \wedge \vec{B}
$$

Blood consists of plasma, red blood cells, white blood cells and platelets. It has been considered as a Newtonian fluid by several researchers (Haik 2001, Tzirtzilakis and Loukopoulos 2005, Tao and Huang 2011, Mohammed 2016). In this study, the blood is considered to be Newtonian, homogeneous and electrically conducting. The thermo-physical properties of the blood and the aorta tissue are assumed to be constant; they are given in Table 1. For the considered configuration (Fig. 1), the boundary conditions are given by:

- At the Aorta inlet, the blood velocity is imposed: $\mathrm{v}=10 \mathrm{~cm} / \mathrm{s}$ and $\mathrm{v}=20 \mathrm{~cm} / \mathrm{s}$ corresponding to the laminar flow with a Reynolds number respectively equal to $\operatorname{Re}=604$ and $\operatorname{Re}=1208$.

- The inlet pressure is considered to be the systolic pressure.

- At the aorta outlet, the fluid flow is considered to be fully developed thus: 


$$
(\partial p / \partial x)=0 ;(\partial u / \partial x)=0 ;(\partial v / \partial x)=0
$$

The non-slip conditions for velocity are applied to the inner wall of the aorta. The magnetic field $\mathrm{B}_{0}$ is considered constant over the entire studied domain (aorta tissue and blood).

\section{Numerical simulation procedure}

The aorta is the largest artery in the body. It originates from the left ventricle of the heart and extends to the abdomen, where it bifurcates into two smaller arteries. It supplies oxygenated blood to all parts of the body via the circulation, except for the functional circulation of the lung (pulmonary artery). It is divided into three sections: the ascending aorta, the arch of the aorta and the descending aorta. The aortic arch is the section that looks like an inverted $U$ shape. The studied aorta has been considered with an idealized geometry where the cross-sections are considered to be circular; the geometry is illustrated in Fig. 1. Fluent software (2006), which is based on the finite volume method, has been used to solve the governing equations (Eqs. 13 to 18). The pressure-based solver has been chosen with the implicit formulation method and the standard pressure interpolation scheme. The second-order upwind scheme has been adopted for the discretization of momentum and energy equations, this scheme is more accurate than the firstorder upwind. SIMPLE algorithm has been introduced for the pressure-velocity coupling.

\begin{tabular}{|l|l|l|l|}
\hline Material & Units & Blood & $\begin{array}{l}\text { Aortic } \\
\text { tissue }\end{array}$ \\
\hline Density & $\mathrm{Kg} / \mathrm{m}^{3}$ & 1050 & 1102 \\
\hline $\begin{array}{l}\text { Dynamic } \\
\text { viscosity }\end{array}$ & $\mathrm{Kg} / \mathrm{ms}$ & 0.00365 & 3306 \\
\hline $\begin{array}{l}\text { Specific } \\
\text { heat }\end{array}$ & $\mathrm{J} / \mathrm{kgK}$ & 3617 & 0.46 \\
\hline $\begin{array}{l}\text { Thermal } \\
\text { conductivit } \\
\mathrm{y}\end{array}$ & $\mathrm{W} / \mathrm{mK}$ & 0.52 & 0.261 \\
\hline $\begin{array}{l}\text { Electrical } \\
\text { conductivit } \\
\mathrm{y}\end{array}$ & $1 / \mathrm{m} \mathrm{mm}$. & 0.65 & 0.0018 \\
\hline $\begin{array}{l}\text { Magnetic } \\
\text { Permeabilit } \\
\mathrm{y}\end{array}$ & $\mathrm{h} / \mathrm{m}$ & 0.0012 & \\
\hline
\end{tabular}

Table 1. Thermo-physical properties of blood and the aorta tissue given by IT'IS (2017) and (IFAC) 


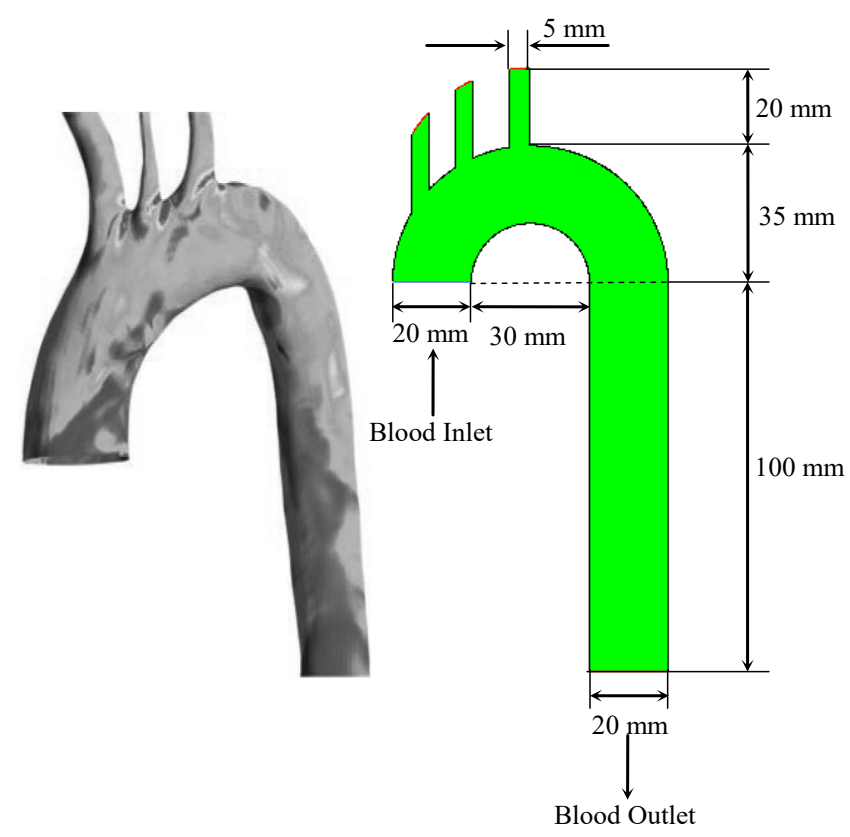

Fig. 1. The geometry of the studied Aorta

In order to choose the most appropriate mesh, a grid independence study was conducted. The independence on mesh size is evaluated through low variation of skin friction coefficient. For that purpose, five different meshes generated by Gambit mesh generator software have been tested; their details are mentioned in Table 2. It can be seen that geometries with mesh 1 to 3 are not suitable because the effect of the grid size on the results is clear. However, the mesh 4 and 5 are acceptable due to the low variation of skin friction coefficient. Thus the best choice for our numerical study is grid 4 which has the lowest number of elements. Detail view of the chosen surface grid is shown in Fig. 2.

\begin{tabular}{|l|l|l|}
\hline Mesh & $\begin{array}{l}\text { Number of } \\
\text { elements }\end{array}$ & $\begin{array}{l}\text { Skin friction } \\
\text { coefficient }\end{array}$ \\
\hline Mesh 1 & 4189 & 0.0331581 \\
\hline Mesh 2 & 6284 & 0.0360573 \\
\hline Mesh 3 & 7028 & 0.0385712 \\
\hline Mesh 4 & 8189 & 0.0395305 \\
\hline Mesh 5 & 11289 & 0.0395304 \\
\hline
\end{tabular}

Table 2. Details of the different tested meshes and corresponding skin friction coefficient

A simulation was performed for blood circulation in the aorta for both cases, with and without external magnetic fields (EMF). Some typical results have been presented in order to study the effect of an applied EMF on blood behaviour. 


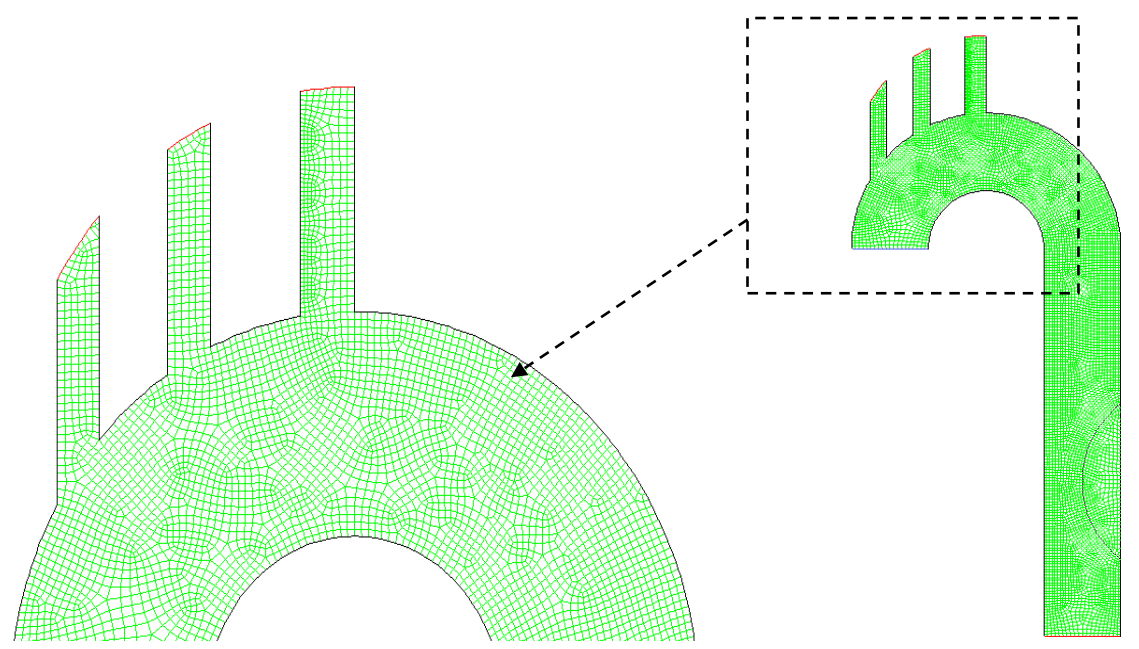

Fig. 2. Details view of the chosen mesh

\section{Results and discussions}

\subsection{External magnetic field effects}

Fig. 3 shows the contours of pressure in the aorta with and without an EMF. The inlet systolic pressure and velocity have been taken successively equal to $120 \mathrm{mmHg}$ and $20 \mathrm{~cm} / \mathrm{s}$ (corresponding to a Reynolds number of 1208). It can be clearly noticed that under the effect of the EMF, the pressure is largely affected. The pressure increases slightly in the ascending part of the aorta and the pressure gradient increases greatly in its descending part.
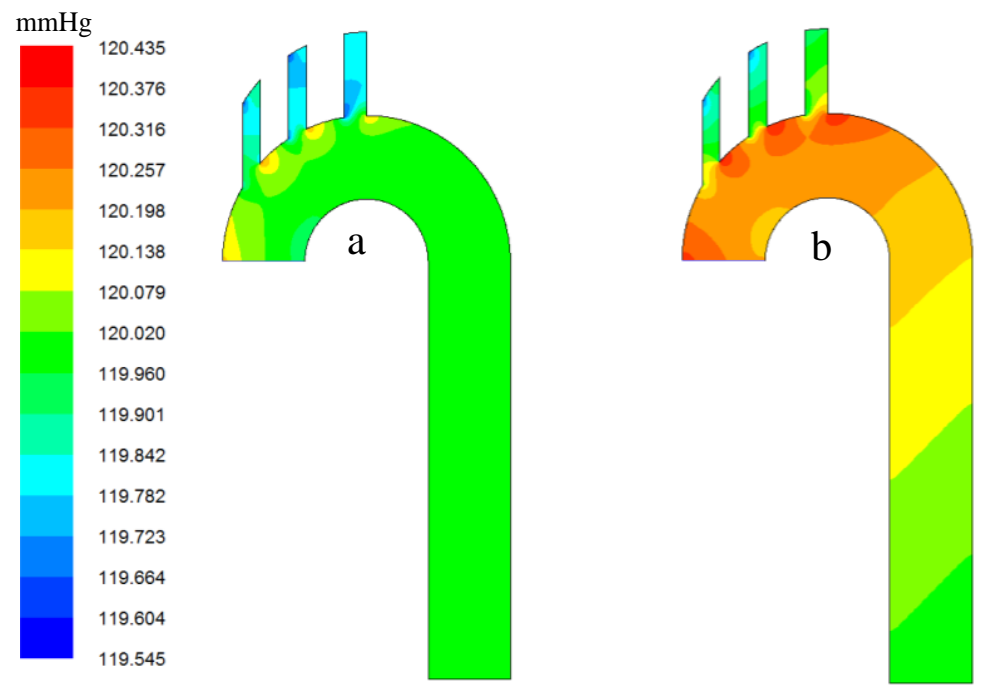

Fig. 3. Contours of systolic pressure in the Aorta. (a) Without EMF; (b) With EMF

For the velocity contours (Fig. 4), it is observed that the velocity distribution in the descending part of the aorta becomes uniform and weaker after the application of EMF. Note that 
the uniformity of the velocity under an EMF has been obtained experimentally by Tao and Huang (2011) by controlling the blood viscosity.
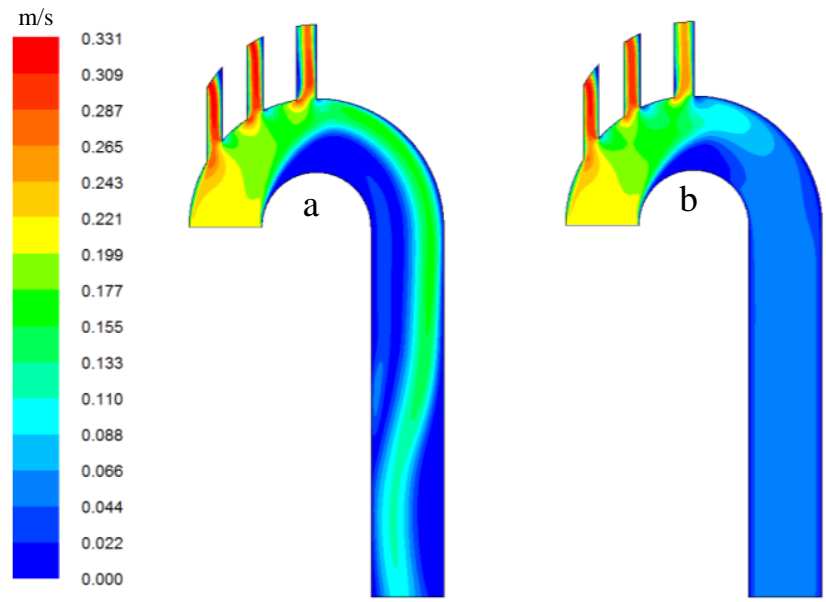

Fig. 4. Velocity contours in the human Aorta. (a) Without EMF; (b) With EMF

On the other hand, the development of the recirculation zone of blood is also affected in the area of the arch of the aorta. Fig. 5 shows that this area has shrunk considerably. From these results, we can say that the EMF allows minimizing the flow instabilities due to the geometrical singularities of the aorta (especially in the region of the arch of the aorta).
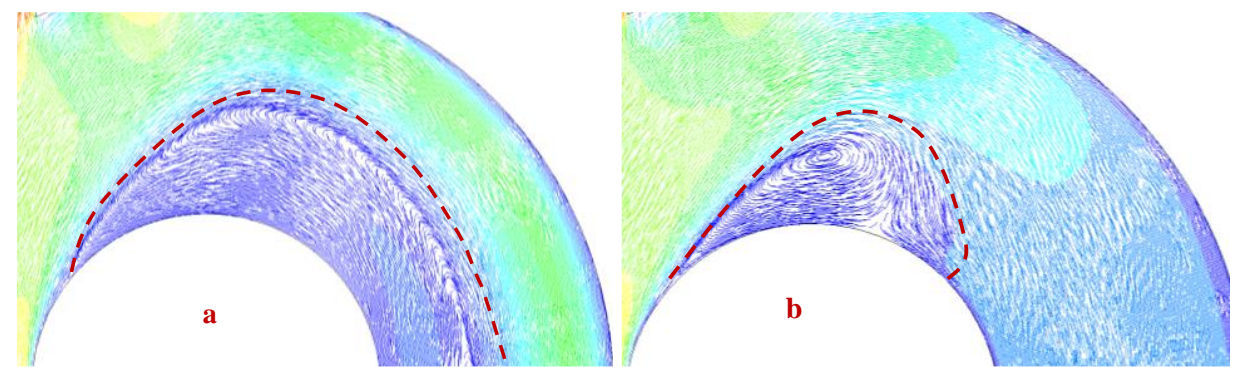

Fig. 5. Development of the blood stagnation zone in the arch of the Aorta. (a) Without EMF; (b) With EMF

\subsection{The atherosclerosis effects}

In order to control blood flow in the aorta, we are interested in the pathological case of atherosclerosis, semicircular shape, on the descending part of the aorta. Fig. 6 shows that the presence of this deposit decreases blood pressure. Since the chosen study model (aorta) has small dimensions, the pressure variation is weak; for this reason we are interested in calculating the pressure gradient that describes the direction and the rate of pressure changes around a particular location. Table 3 shows the different pressure gradients in the aorta with and without atherosclerosis, where we can notice that the decrease of the pressure gradient is about $43 \%$.

\begin{tabular}{|l|l|}
\hline Pressure gradients & $\Delta \mathrm{P} / \mathrm{L}$ \\
\hline
\end{tabular}




\begin{tabular}{|l|l|}
\hline $\mathrm{mm} \mathrm{Hg} / \mathrm{m}$ & \\
\hline Without atherosclerosis & 0.388 \\
\hline With atherosclerosis & 0.222 \\
\hline Percentage of decrease \% & 42.78 \\
\hline
\end{tabular}

Table 3. Pressure gradients in the Aorta with and without atherosclerosis
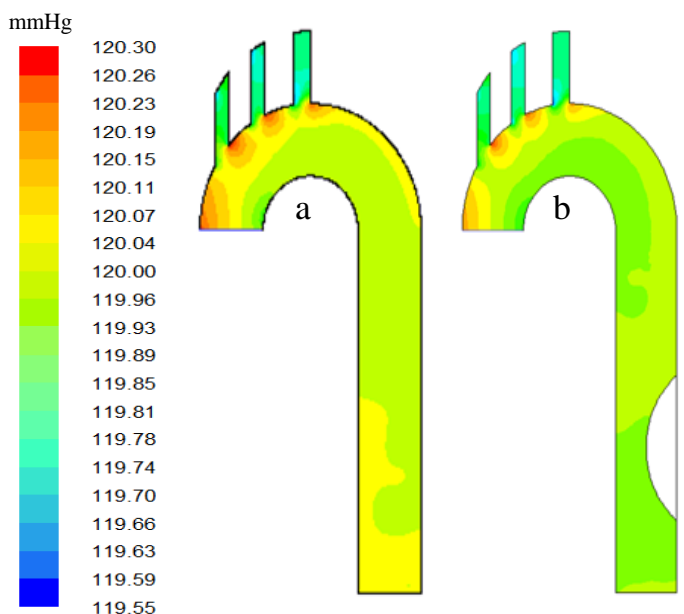

Fig. 6. Contours of systolic pressure in the Aorta. (a) Without atherosclerosis; (b) With atherosclerosis

The presence of atherosclerosis also affects the distribution of local velocity in the aorta (Fig. 7). It increases the blood velocity in the surrounding area and creates recirculation zones (Fig. 8).
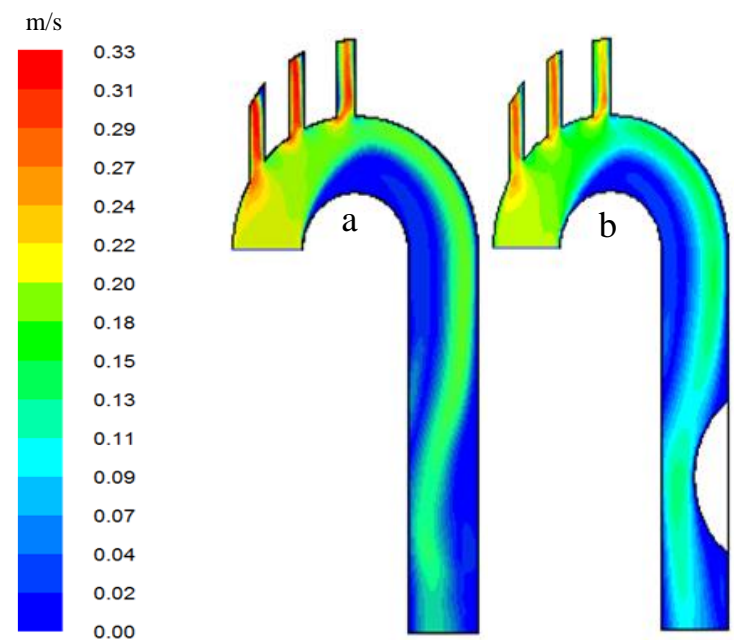

Fig. 7. Contours of velocities in the Aorta. (a) Without atherosclerosis; (b) With atherosclerosis 
This results firstly in the reduction of the section of the aorta, because of the deposit, and secondly, it is considered as an obstruction to the passage of blood. This has the effect of reducing the pressure and the blood flow. Blood circulation becomes difficult and cells lack oxygen and nutrients.
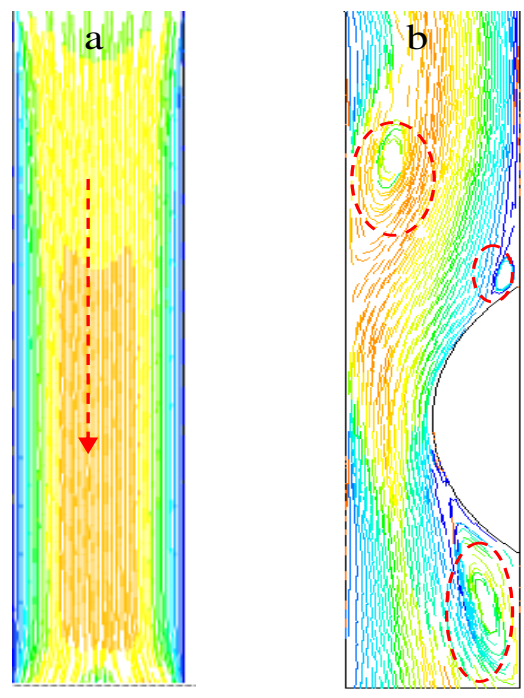

Fig. 8. Streamlines and recirculation zones in the descending part of the Aorta. (a) Without atherosclerosis; (b) With atherosclerosis

\subsection{Flow control using EMF}

In order to control the flow in the aorta with atherosclerosis, an EMF of intensity equal to $3 \mathrm{~T}$ has been applied in both (x) and (y) directions for 60 seconds. The (x) direction corresponds to the flow direction and the (y) one is transverse to the direction of flow. The aim of this control study is to facilitate the blood passage around the atherosclerosis (in the descending part of the aorta). The inlet systolic pressure and velocity have been taken successively equal to $120 \mathrm{mmHg}$ and 10 $\mathrm{cm} / \mathrm{s}$ (corresponding to a Reynolds number of 604). Fig. 9 shows systolic pressure comparison between three studied cases: without an EMF, with an EMF applied in the (x) direction and with an EMF applied in the (y). The results show that only the EMF applied in the direction of the flow that created a pressure gradient, which facilitates the blood flow around the area of atherosclerosis, compared to the other cases (Fig. 10). The relatively uniform velocity contours are obtained when the EMF has been applied in (y) direction (Fig. $10 \mathrm{c}$ ). The morphology of the recirculation zone has also been significantly modified. 

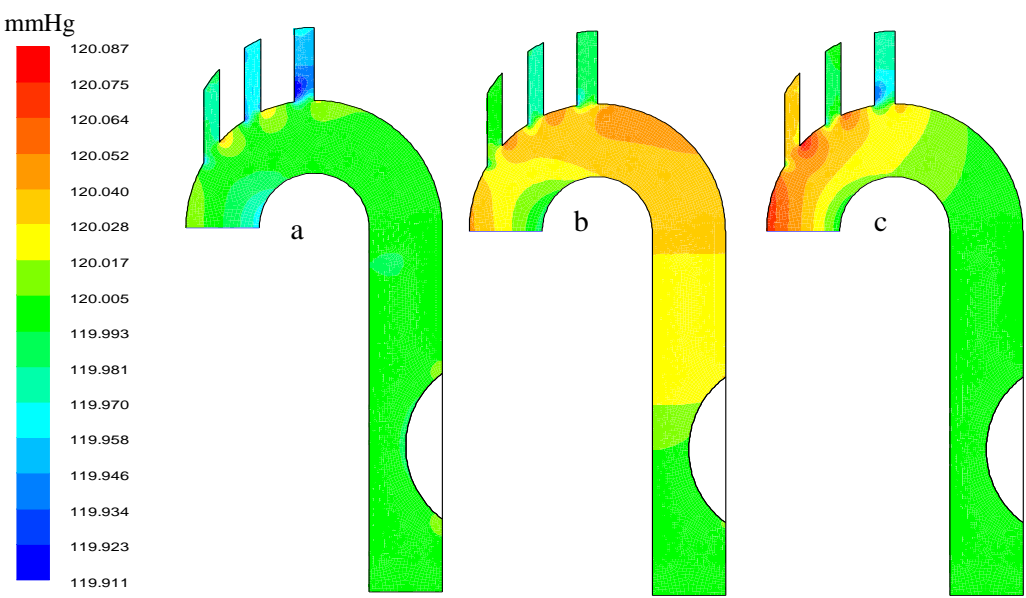

Fig. 9. Contours of systolic pressure in the Aorta. (a) Without EMF; (b) EMF $B_{0_{-} x}$ component;

(c) EMF $B_{0 \_y}$ component
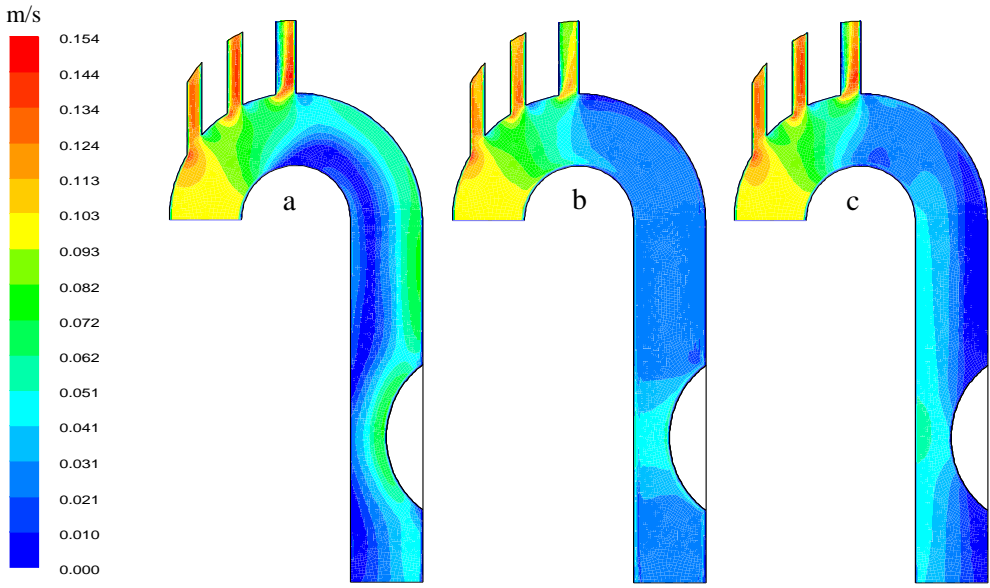

Fig. 10. Velocity contours in the Aorta. (a) Without EMF; (b) EMF $B_{0_{-} x}$ component; (c) EMF $\mathrm{B}_{0 \_\mathrm{y}}$ component

Fig. 11 shows that when applying an EMF in the transverse direction of the flow, the recirculation zones in the front of atherosclerosis disappear but small areas have been created upstream and downstream of the atherosclerosis face. However, when the direction of the EMF is parallel to the flow direction, the recirculation zones disappear completely and the streamlines are, therefore, similar to the aorta without atherosclerosis (Fig. 8a). It can be concluded that, The EMF direction parallel to the flow direction has a more significant effect on the flow than that in the case of a transverse direction of the EMF. 

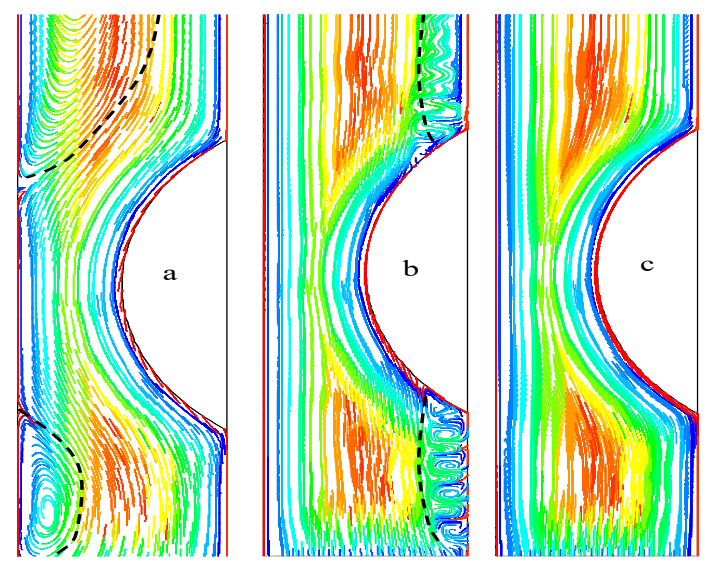

Fig. 11. Streamlines in the Aorta. (a) Without EMF; (b) EMF $B_{0_{-} x}$ component; (c) EMF $B_{0_{-} y}$ component

The effect of the EMF intensity on pressure and velocity distributions in the aorta has been also studied in order to control the blood flow. Fig. 12 shows the systolic pressure contours in the aorta for different EMF intensities. A significant increase in the pressure magnitude is observed due to the increase in the EMF intensity. The largest pressure gradient along the aorta is obtained for the highest intensity values. The more uniform velocity in the aorta is also obtained for highest intensity values (Fig. 13).
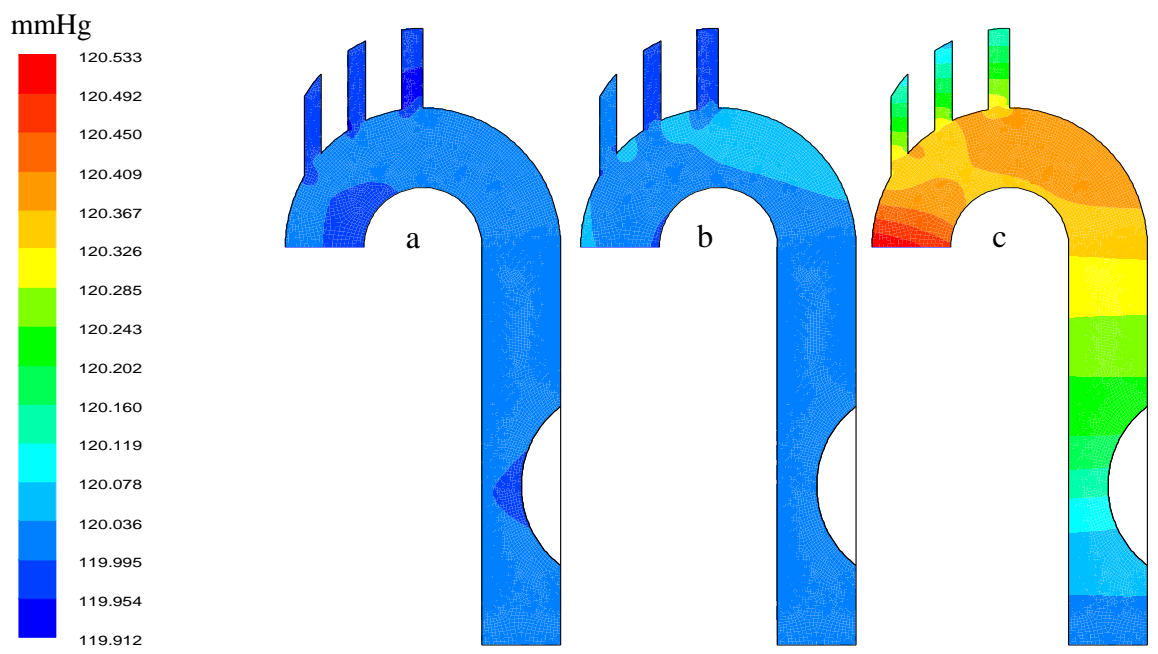

Fig. 12. Contours of systolic pressure in the Aorta, inlet velocity of $10 \mathrm{~cm} / \mathrm{s}(\operatorname{Re}=604)$. (a) $B_{0_{-} y}$ $=1 \mathrm{~T}$; (b) $\mathrm{B}_{0 \_\mathrm{y}}=3 \mathrm{~T}$ and (c) B0_y $=5 \mathrm{~T}$

The recirculation zone is significantly affected by the EMF intensity; this zone can disappear completely for the highest intensity values (Fig. $14 \mathrm{c}$ and d). On the other hand, it has been found that for the highest EMF intensity values, the velocity in the cross-section A-A (in the ascending part of the aorta, where the recirculation zone occurs (Fig. 14 a)) becomes more uniform except near the aorta wall where a strong velocity gradient is obtained as shown in Fig.15. The shear stress profile in the descending zone of the Aorta (on the side of the Atherosclerosis) is shown in 
Fig. 16. It may be noted that, the most uniform variations of the shear stress are obtained for the highest EMF intensity values, thus giving the most regular streamlines.
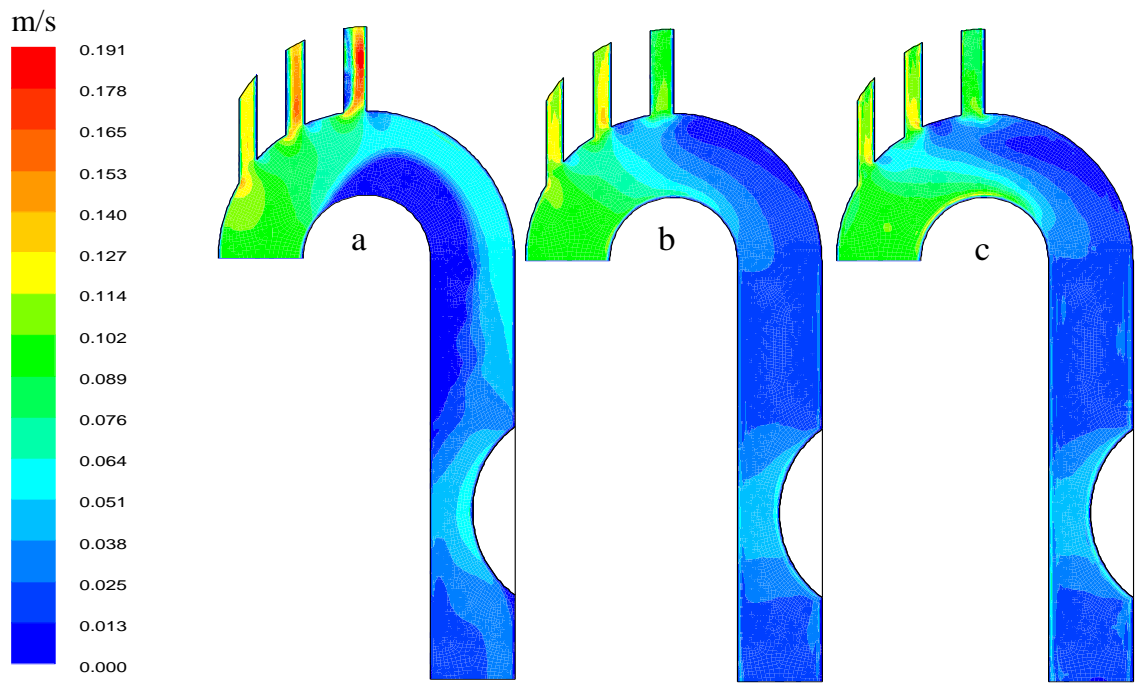

Fig. 13. Velocity contours in the Aorta, inlet velocity of $10 \mathrm{~cm} / \mathrm{s}(\operatorname{Re}=604)$. (a) $\mathrm{B}_{0 \_} \mathrm{y}=1 \mathrm{~T}$; (b) $\mathrm{B}_{0 \_\mathrm{y}}=3 \mathrm{~T}$; (c) $\mathrm{B}_{0 \_\mathrm{y}}=5 \mathrm{~T}$

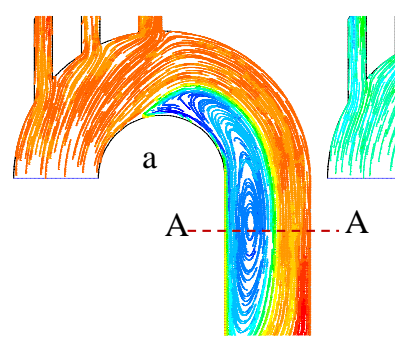

b
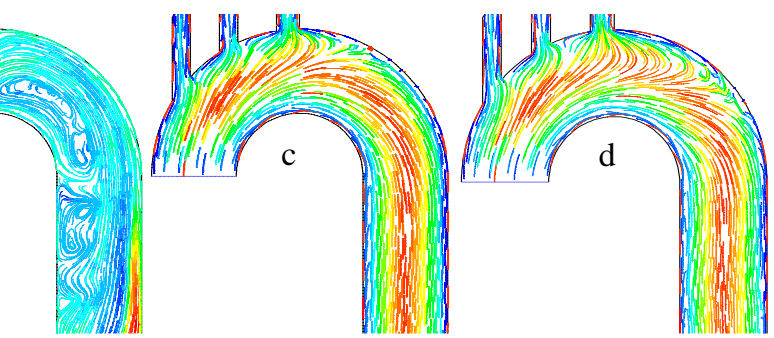

Fig. 14. Morphology of the recirculation zone. (a) Without EMF; (b) $\mathrm{B}_{0_{-} \mathrm{y}}=1 \mathrm{~T}$; (c) $\mathrm{B}_{0_{-} \mathrm{y}}=3 \mathrm{~T}$; (d) $\mathrm{B}_{0-\mathrm{y}}=5 \mathrm{~T}$ 


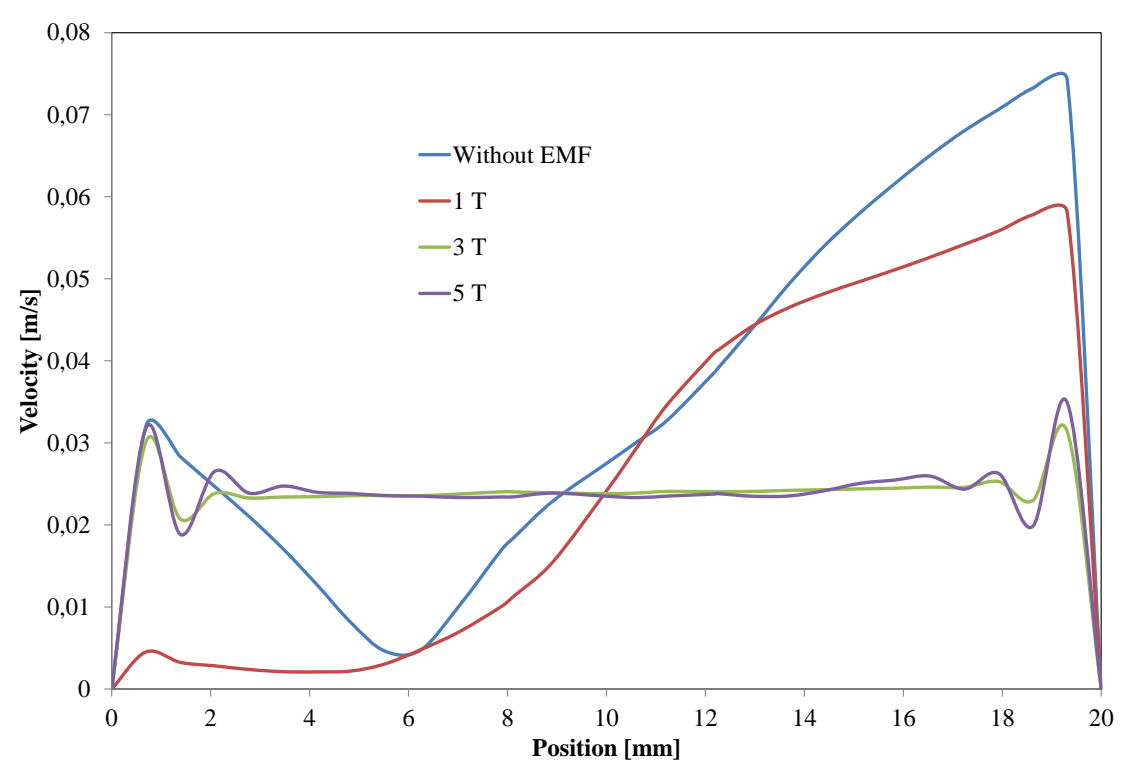

Fig. 15. Cross-section (A-A) velocity profile for different EMF density

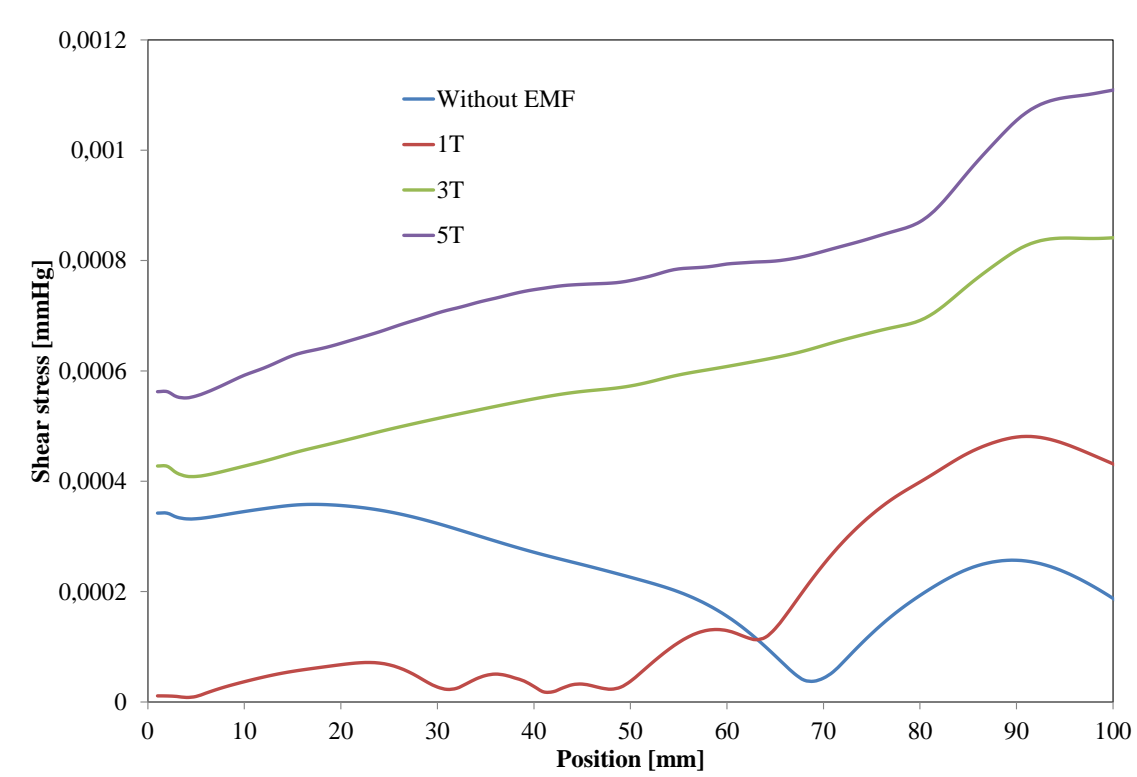

Fig. 16. Wall shear stress in the descending zone of the Aorta (on the side of the Atherosclerosis)

\section{Conclusions}

In this analysis, a numerical investigation of the blood flow in the aorta under an external magnetic field (EMF) was performed. We can note that, by applying an EMF, the blood flow can be controlled in order to obtain a good circulation throughout the aorta, especially around the atherosclerosis. From this study, the following conclusions have been obtained: 
- By applying an EMF, the velocity in the descending part of the aorta has been reduced and becomes more uniform. The development of the recirculation zone of blood is also affected; this recirculation zone has been decreased significantly in the arch of the aorta.

- The blood flow was controlled by the EMF direction. When the EMF was applied in the blood flow direction, a relatively strong pressure gradient was obtained and the blood flow becomes more regular around the atherosclerosis. The recirculation zones disappear completely and the streamlines are, therefore, similar to the aorta without atherosclerosis.

- The blood flow was also controlled by the EMF intensity. The increase in the EMF intensity led to an increase in the pressure magnitude and in the pressure gradient along the aorta. The more uniform velocity in the aorta is also obtained for highest intensity values and the recirculation zone disappears completely. The wall shear stress increases with magnetic field strength.

From these results, we can say that the blood flow in the human aorta can be controlled by applying an EMF. It allows minimizing the flow instabilities due to the geometrical singularities of the aorta.

For further improvements, we suggest studying the non-Newtonian case of the blood with a pulsating input velocity corresponding to the heart rate.

\section{References}

Aggoune M, Aggoune N, Bensedira N, Bouhantala A, and Belkbir A (2017). Simulation of a 2D Magnetic Drug Targeting Model. International Journal of Civil and Structural Engineering 4, 345-355.

Alghamdi MS and El-Ghazaly N (2012). Effects of Exposure to Electromagnetic Field on Some Hematological Parameters in Mice. Open Journal of Medicinal Chemistry 2, 30-42.

Ali Shah N, Vieru D, and Fetecau C (2016). Effects of the fractional order and magnetic field on the blood flow in cylindrical domains. Journal of Magnetism and Magnetic Materials 409, 10-19.

Ducreux JP, Guillot Y, Thomas P, Burais N, and Scorretti R (2009, June). Benchmarks for computing induced currents in the human body by ELF electric and magnetic fields. In Proceedings of ELF EMF, Sarajevo, Bosnia and Herzegovina.

Fluent Inc. (2006). Lebanon, New Hampshire, USA.

Frénéa-Robin M, Burais N, Buret F, Haddour N, and Nicolas L (2009, November). Electromagnetic characterization of biological cells. In Proceedings of: International Conference on Electromagnetic Fields, Health and Environment EHE'09, São Paulo, Brazil.

Gmitrov J (2014). Static Magnetic Field Effect on Cardiovascular Regulation: A Review. British Journal of Medicine \& Medical Research 4, 1612-1627.

Gungor H, Akkaya S, Yorukoglu A, Yorukoglu C, Kiter E, Oguz E, Keskin N, and Mete G (2015). Chronic exposure to static magnetic fields from magnetic resonance imaging devices deserves screening for osteoporosis and vitamin D levels: a rat model. International Journal of Environmental Research and Public Health 12, 8919-8932.

Haik Y, Pai V and Chen C (2001). Apparent viscosity of human blood in high static magnetic field. Journal of Magnetism and Magnetic Materials 225, 180-186.

Health Protection Agency, Centre for Radiation, Chemical and Environmental Hazards (2008). Static magnetic fields: report of the independent Advisory Group on Non-ionising Radiation. Health Protection Agency, UK. 
Hoang LH, Scorretti R, Burais N, and Voyer D (2009). Numerical Dosimetry of Induced Phenomena in the Human Body by a Three-Phase Power Line. IEEE Transactions on Magnetics 45(3), 1666-1669.

(IFAC): Institute of applied physics "Nello Carrara", Florence, Italian National Research Council: Dielectric Properties of Body Tissues: http://niremf.ifac.cnr.it/tissprop/htmlclie/htmlclie.php

IT'IS (2017), The Foundation for Research on Information Technologies in Society (IT'IS): https://www.itis.ethz.ch/virtual-population/tissue-properties/database/database-summary/

Kanai S, and Taniguchi N (2012). Efficacy of static magnetic field for pain of adjuvant arthritis rats. Advances in Bioscience and Biotechnology 3, 511-515.

Kenjere S (2008). Numerical analysis of blood flow in realistic arteries subjected to strong nonuniform magnetic fields. International Journal of Heat and Fluid Flow 29, 752-764.

Misra JC and Shit GC (2007). Effect of magnetic field on blood flow through an artery: a numerical model. Computing technologies 12(4), 3-15.

Mohammed A (2016). A Study on Influence of Magnetic Field on Blood. International Archive of Applied Sciences \& Technology 7, 61-65.

Parmar L, Kulshreshtha SB, and Singh DP (2013). The role of magnetic field intensity in blood flow through overlapping stenosed artery: A Herschel-Bulkley fluid model. Advances in Applied Science Research 4(6), 318-328.

Sharma MK, Sharma PR, and Nasha V (2013). Pulsatile MHD Arterial Blood Flow in the Presence of Double Stenoses. Journal of Applied Fluid Mechanics 6(3), 331-338.

Sharma S, Singh U, and Katiyar VK (2015). Magnetic field effect on flow parameters of blood along with magnetic particles in a cylindrical tube. Journal of Magnetism and Magnetic Materials 377, 395-401.

Siauve N, Scorretti R, Burais N, Nicolas L, and Nicolas A (2003). Electromagnetic Fields and Human Body: a New Challenge for the Electromagnetic Field computation. International journal for computation and mathematics in electrical and electronic engineering 22, 457469.

Tao R and Huang K (2011). Reducing blood viscosity with magnetic fields. Physical Review 84, 001900.

Tzirtzilakis E and Loukopoulos V (2005). Biofluid flow in a channel under the action of a uniform localized magnetic field. Computational Mechanics 36, 360-374.

Yu S and Shang P (2014). A review of bio-effects of static magnetic field on rodent models. Progress in Biophysics and Molecular Biology 114, 14-24. 